\title{
Intermittent Stretching and Osteogenic Differentiation of Bone Marrow Derived Mesenchymal Stem Cells via the p38MAPK- Osterix Signaling Pathway
}

\author{
Wen-lin Xiao Dai-zun Zhang Cun-hui Fan Bao-jun Yu \\ The Key Laboratory of Oral Medicine, Shandong Province Education Department; Department of \\ Stomatology, Affiliated Hospital of Qingdao University; Qingdao, People's Republic of China
}

\section{Key Words}

Intermittent stretching force $\cdot$ BMMSCs•Osteogenic differentiation • p38MAPK signal pathway - Osterix

\begin{abstract}
Aims: The relationship between the p38MAPK signaling pathway and osterix in osteogenic differentiation of BMMSCs subjected to intermittent stretching was investigated. Methods: BMMSCs derived from C57BL/6J mice were divided into the following groups: 1) control, 2) stretch, and 3) SB203580+stretch (SB203580 is a p38MAPK signal pathway inhibitor). BMMSCs were exposed to an intermittent mechanical strain of $0.8 \%(8000 \mu$ strain) at $0.5 \mathrm{~Hz}$, twice a day for 30 min each application. BMMSCs were harvested on days 1, 3, and 5 post-treatment. The expression of ALP, COL I, OCN, and osterix mRNA was assessed utilizing RT-PCR while the expression of P-p38MAPK and osterix protein was assessed by Western blot analysis. The osterix gene in mouse BMMSCs was knocked down using RNAi technology and its protein expression was also assessed by Western blot. RT-PCR was used to detect ALP, COL I, and OCN mRNA expression. Results: Intermittent stretching was found to promote expression of ALP, COL I, OCN, and osterix mRNA. Silencing the osterix gene was found to reduce levels of ALP, COL I, and OCN mRNA. Western blot analysis demonstrated that the levels of osterix and P-p38MAPK proteins in the stretch group were significantly higher than in the control group $(P<0.05)$. There was less expression of ALP, COL I, OCN, and osterix mRNA in the SB203580+stretch group than in the control and stretch groups. Conclusions: Data demonstrate that intermittent stretching promotes osteogenic differentiation of BMMSCs, and the p38MAPK-osterix pathway has an important role in the control of osteogenesisrelated gene expression.
\end{abstract}




\section{Introduction}

Bone marrow mesenchymal stem cells (BMMSCs) are multipotent stem cells with the ability to undergo tri-lineage differentiation. Compared to other types of cells, they are one of the most sensitive to mechanical stretching. Previous studies have demonstrated that mechanical stretching of BMMSCs can induce osteogenic differentiation. [1]. The p38MAPK signaling pathway plays a crucial role in the inflammatory reaction, regulation of the cell cycle, and cellular differentiation. Studies have demonstrated that the p38MAPK signaling pathway is related to osteogenic differentiation of BMMSCs [2-6]. Osterix is a recently discovered transcription factor related to bone formation as well as osteogenic differentiation. It contains a zinc finger motif that is specifically expressed in osteoblasts [7, 8] Studies have shown that mechanical stimulation can induce high levels of osterix mRNA expression [9-11]. However, current studies have not produced information regarding the relationship between the p38MAPK signaling pathway and osterix during osteogenic differentiation of BMMSCs induced by intermittent stretching. The purpose of this study was to investigate the relationship between the p38MAPK signaling pathway and osterix during osteogenic differentiation of BMMSCs induced by intermittent stretching.

\section{Materials and Methods}

\section{BMMSC isolation and culture}

BMMSCs were isolated from the femurs and tibias of 30-day-old male C57BL/6J mice (Beijing HuaFukang Experimental Animal Center, Beijing, China) as previously described [12, 13]. The BM was washed with 2\% FBS in PBS (Gibco, Grand Island, NY). The cell suspension was subsequently passed through syringes of 20,22, and $25 \mathrm{~g}$ in a sequential manner Cell suspensions were collected in $15 \mathrm{ml}$ centrifuge tubes and centrifuged at $400 \mathrm{~g}$ for $5 \mathrm{~min}$. The cell pellets were resuspended with a-MEM (Hyclone, Logan, UT) containing $15 \%$ FBS. The cells were then seeded in a $25 \mathrm{~cm}^{2}$ culture flask at a density of $1.2 \times 10^{6}$ cells $/ \mathrm{ml}$ and incubated at $37^{\circ} \mathrm{C}$ in a humidified incubator in $95 \%$ air and $5 \% \mathrm{CO}_{2}$ for $3 \mathrm{~h}$. The media was changed once, then every $12 \mathrm{~h}$ through the first $48 \mathrm{~h}$ and every 3 days thereafter. After reaching $80-90 \%$ confluence, cells were subcultured or plated for subsequent experiments. The cells were identified by detecting the expression of CD44 (+), CD105 (+), Sca-1 (+), CD11b (-), CD34 (-), and CD45 (-) via flow cytometry.

BMMSCs were found to differentiate into adipocytes and osteoblasts when cultured in adipogenic or osteogenic media, respectively. Oil red 0 staining and Alizarin Red-S staining were used to assess the adipogenic and osteogenic differentiation of BMMSCs. To evaluate the potential of BMMSCs to undergo osteogenic differentiation, BMMSCs (p2) were seeded at a density of $1 \times 10^{5}$ cells per well in 6-well plates. After reaching $\sim 80 \%$ confluence, BMMSCs were cultured in osteogenic media (Sigma, Santa Clara, CA). The $\alpha$-MEM was supplemented with $2 \%$ FBS, $100 \mathrm{nM}$ dexamethasone, $50 \mathrm{pg} / \mathrm{ml}$ ascorbic acid and $5 \mathrm{mM}$ $\beta$-glycerophosphate. The media were changed every 2 days. Mineralization of BMMSCs was determined by Alizarin Red-S staining at day 28 post-treatment. BMMSCs were washed with PBS and fixed with $4 \%$ paraformaldehyde in PBS for 15 min. The fixed cells were stained with Alizarin Red-S solution (Sigma, Santa Clara, CA) for 30 min followed by washing with distilled water. Calcified nodules, which appeared bright red in color, were photographed using inverted phase contrast microscopy (Olympus IX50, Olympus, Japan). For induction of adipocyte differentiation, BMMSCs (p2) were seeded at a density of $1 \times 10^{5}$ cells per well in 6-well plates. After reaching $80 \%$ confluence, the BMMSCs were cultured in adipogenic media (Sigma, Santa Clara, CA). The $\alpha$-MEM was supplemented with 2\% FBS, $100 \mu \mathrm{M}$ indomethacin, $0.1 \mu \mathrm{M}$ dexamethasone, $0.5 \mathrm{mM}$ 3-isobutyl-1-methylxanthine (IBMX), and $10 \mu \mathrm{g} / \mathrm{ml}$ insulin for about 20 days. The media were changed every 2 days. Then, the adipogenic cultures were fixed in $4 \%$ paraformaldehyde for $30 \mathrm{~min}$ and stained with fresh Oil Red 0 solution (Sigma, Santa Clara, CA) for 15 min to detect adipocyte formation under inverted phase contrast microscopy (Olympus IX50, Olympus, Japan). BMMSCs from passages 2 and 3 were used for subsequent experiments.

Intermittent stretching of mouse BMMSCS

BMMSCs (p2) were trypsinized with $0.25 \%$ trypsin (Gibco, Grand Island, NY) and then seeded onto 6-well silicone rubber plates at a density of $1 \times 10^{5}$ cells $/ \mathrm{cm}^{2}$. The cells were cultured for $48 \mathrm{~h}$ allowing 


\section{Cellular Physiology Cell Physiol Biochem 2015;36:1015-1025 \begin{tabular}{l|l} 
DOI: 10.1159/000430275 & $\begin{array}{l}\text { O 2015 S. Karger AG, Basel } \\
\text { www.karger.com/cpb }\end{array}$ \\
\hline
\end{tabular}

them to adhere to the 6 -well silicone rubber plates until an $80-90 \%$ confluence was reached. Intermittent mechanical stretching of the BMMSCs was accomplished by cell loading with the FX-4000T ${ }^{\mathrm{TM}}$ Flexercell Tension Plus ${ }^{\mathrm{TM}}$ unit (Flexcell International Corporation, Hillsborough, NC) at $0.5 \mathrm{~Hz}$, elongation at $0.8 \%$, twice each day for $30 \mathrm{~min}$ each time.

Blocking the p38MAPK signaling pathway using SB203580

The BMMSCs were randomly divided into three experimental groups including control, stretch, and SB203580+stretch. The cells in the SB203580+stretch group were pre-treated with SB203580 (a p38MAPK pathway-specific inhibitor, Cell Signaling, Danvers, MA) (10 $\mathrm{mol} / \mathrm{l})$ for $1 \mathrm{~h}$ at $37^{\circ} \mathrm{C}$, before mechanical stimulation. The entire experimental process was performed using the instructions provided by the manufacturer of the SB203580. The total RNA and cell lysates from the BMMSCs were harvested after 1, 3 , and 5 days of stretch stimulation, in separate groups. The mRNA expression levels of ALP, Col I, OCN, and osterix were assessed using a RT- PCR kit (Takara, Dalian, China) and the expression of the p38MAPK, P-p38MAPK, and osterix proteins were assessed using Western blot analysis.

siRNA-osterix/lipofectamine2000 composite construction and cell transfection

The target osterix mRNA sequence (mouse; NM_130458), was searched in the NCBI GenBank Database and small interfering RNAs (siRNAs) against mouse osterix were designed and synthesized using GenePharma Biological Company (Shanghai, China). The optimal sequence was selected: 5'-CAAGGCAGUUGGCAAUAGUTT-3'; 5'-ACUAUUGCCAACUGCCUUGTT-3'. The siRNA negative control did not recognize any known homology of mouse genes. BMMSCs were transfected with siRNA using Lipofectamine after reaching approximately 80\% confluence, in accordance with the manufacturer's instructions. The BMMSCs were cultured for another $24 \mathrm{~h}$ before intermittent stretching. Instead of target siRNA, the negative control group cells were transfected with non-silencing siRNA. The total RNA and cell lysates of the BMMSCs were harvested after 1 day of stretch stimulation. The expression of ALP, Col I, and OCN mRNAs was assessed using real-time PCR. The expression of osterix protein was assessed using Western blot analysis.

\section{Real-time PCR analysis}

Total RNA from the cells was isolated and the cDNA was reversed-transcribed from the mRNA Realtime PCR was performed as described using a LightCycler ${ }^{\circledR} 480$ II system with SYBR Green Premix Ex $\mathrm{Taq}^{\mathrm{TM}}$ (Takara, Dalian, China) according to the manufacturer's instructions. The settings were as follows: denaturation at $95^{\circ} \mathrm{C}(10 \mathrm{~s}) ; 50$ cycles at $95^{\circ} \mathrm{C}(10 \mathrm{~s})$, and $60^{\circ} \mathrm{C}(30 \mathrm{~s})$. GAPDH served as the internal control. The data were analyzed using the comparative Ct $\left(2^{-\Delta \Delta C t}\right)$ method and are expressed as a fold change with respect to the control. All experiments were performed in triplicate. Primer sequences of the genes are shown in Table 1.

\section{Western blot analysis}

The total protein from the BMMSCs was extracted using a cell lysate isolation kit (Qiagen, Valencia, CA) and the protein mixture was centrifuged to remove cellular debris. $20 \mu \mathrm{g}$ of total protein was subjected to $10 \%$ SDS-PAGE and electrotransferred to PVDF membranes. The PVDF membranes were blocked for 2 $\mathrm{h}$ at room temperature using 5\% powdered milk in TBST. The PVDF membranes were allowed to incubate with the following primary antibodies at a dilution of 1:1000: p38MAPK polyclonal antibody, P-p38MAPK polyclonal antibody (Thr180/Tyr182), and osterix polyclonal antibody (all antibodies from Cell Signaling Technology, Danvers, MA). Proteins were then incubated with a peroxidase-conjugated secondary antibody

Table 1. Primer Sequences

\begin{tabular}{lll}
\hline Gene names & Forward $\left(5^{\prime} \rightarrow 3^{\prime}\right)$ & Reverse $\left(5^{\prime} \rightarrow 3^{\prime}\right)$ \\
\hline ALP & ATGGTAACGGGCCTGGCTACA & AGTTCTGCTCATGGACGCCGT \\
OCN & CCTGGCAGGTGCAAAGCCCA & GGGGGCTGGGGCTCCAAGT \\
COL I & CTGCCCAGAAGAATATGTATCACC & GAAGCAAAGTTTCCTCCAAGACC \\
OSX & GATGGCGTCCTCTCTGCTT & TATGGCTTCTTTGTGCCTCC \\
GAPDH & GGCACAGTCAAGGCTGAGAATG & ATGGTGGTGAAGACGCCAGTA \\
\hline
\end{tabular}




\section{Cellular Physiology Cell Physiol Biochem 2015;36:1015-1025 \begin{tabular}{ll|l} 
and Biochemistry 101159/000430275 & $\begin{array}{l}\text { O 2015 S. Karger AG, Basel } \\
\text { www.karger.com/cpb }\end{array}$ \\
\cline { 2 - 3 }
\end{tabular}

for $45 \mathrm{~min}$ at room temperature. After each round of incubation, the membranes were washed in TBST for $20 \mathrm{~min}$. Protein bands were detected, and the relative intensities were analyzed and compared to their respective controls using Image J software.

\section{Statistical analysis}

Each experiment was performed three times in totality. All data are presented as mean \pm SD and significant differences were determined using one-way ANOVA. $P<0.05$ was considered statistically significant.

\section{Ethics statement}

All experiments involving the use of animals were performed in compliance with Provisions and the General Recommendation of Chinese Experimental Animals Administration Legislation and approved by the Ethics Committee of the Affiliated Hospital of Qingdao University (Qingdao, China).

\section{Results}

\section{BMMSCs were cultured and identified}

The primary cultures of C57BL/6J mouse BMMSCs reached 80-90\% confluence in 10 days. Cells were subcultured and designated as passage 1 (p1). The cell morphology of the p2 BMMSCs became consistent and similar to that of typical fibroblasts (Fig. 1). Staining demonstrated the presence of Alizarin Red-S $\mathrm{S}^{+}$mineralized nodules following osteogenic induction of the BMMSCs (Fig. 2A). Further Oil red 0 staining demonstrated the presence of adipocytes following adipogenic induction of the BMMSCs (Fig. 2B) demonstrating the multilineage differentiation potential of BMMSCs. Flow cytometry demonstrated negative cell ratios of CD11b, CD34, and CD 45 to be $5.87,0.43$, and $1.73 \%$, respectively. The percentage of CD44, CD105, and Sca-1 cells within BMMSC population were 99.67, 99.80, and 99.81\%, respectively (Fig. 3). This collectively suggests that the cells isolated and subsequently cultured were indeed BMMSCs and pure enough to meet the requirements [12-14].

Fig. 1. Passage and culturing of BMMSCs in vitro; cell morphology was uniform and fusiform in nature similar to that of fibroblasts.

Fig. 2. A) Results of BMMSCs Alizarin Red-S staining after osteogenic media culturing $(\times 100)$. B) Results of BMMSCs Oil Red 0 staining after adipogenic media $(\times 400)$.
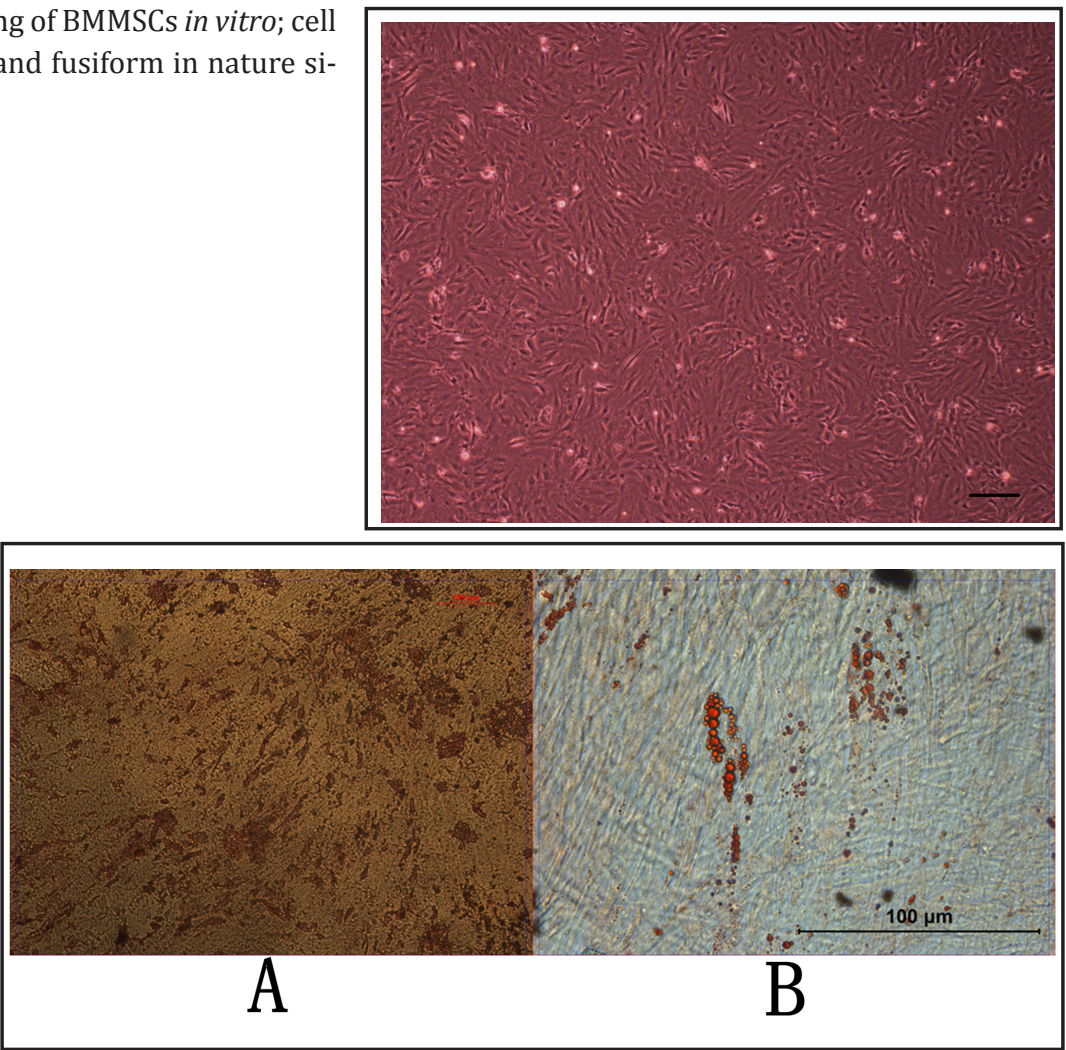
Fig. 3. Expression of cell surface markers in cultured BMMSCs as detected by flow cytometry. The surface marker CD44, CD105, and Sca-1 were positively expressed by $99.67 \%, 99.80 \%$, and $99.81 \%$ of the cells, respectively.

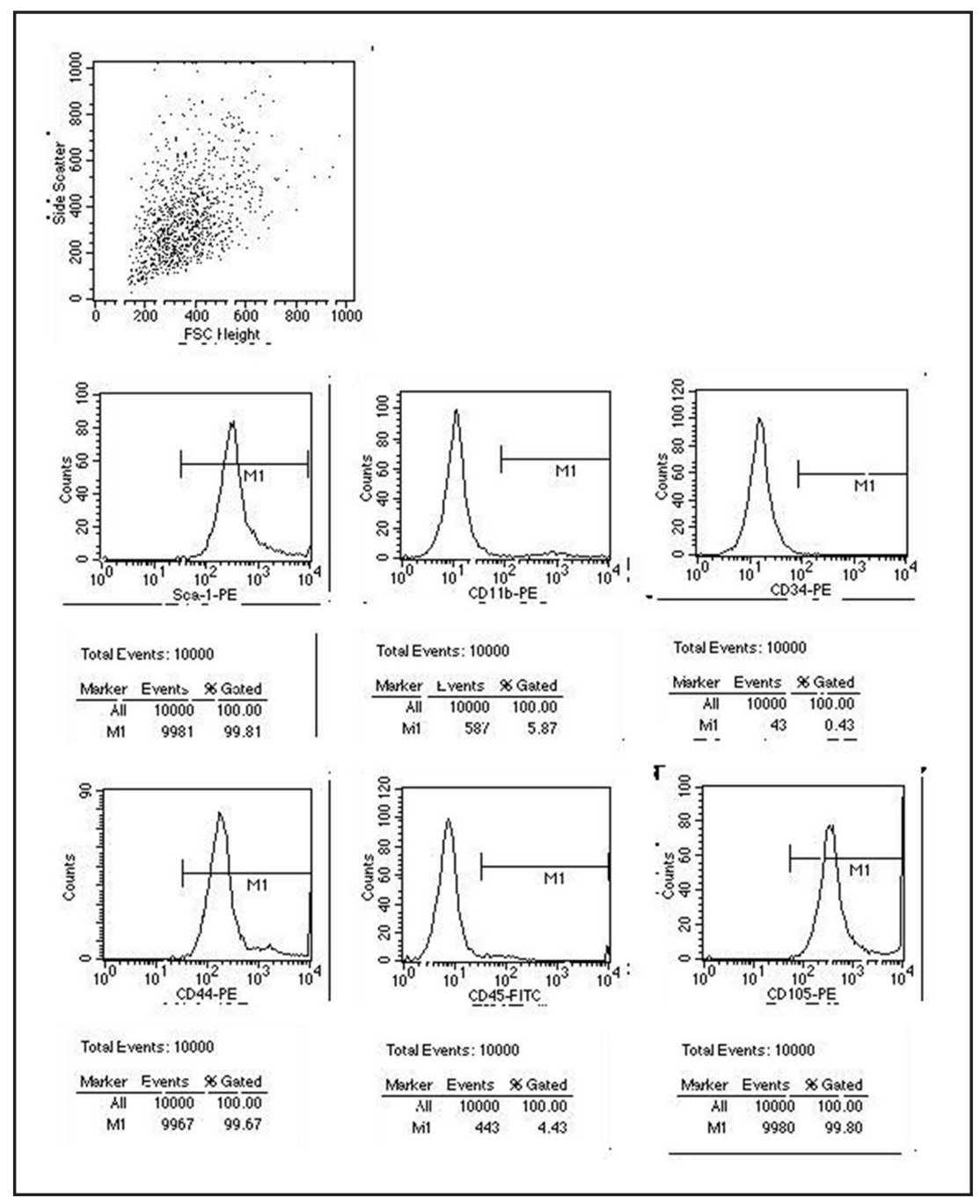

Intermittent stretching induced osteogenic differentiation among BMMSCs

To determine the osteogenic potential of BMMSCs following intermittent stretching, the mRNA expression of certain osteogenesis-related markers including ALP, COL I, OCN, and osterix, were examined. BMMSCs were subjected to intermittent stretching twice daily at $0.5 \mathrm{~Hz}, 0.8 \%$, for $30 \mathrm{~min}$. RT-PCR results demonstrated that the ALP, COL I, OCN, and osterix mRNA levels to be $1.297 \pm 0.175,1.347 \pm 0.126,2.587 \pm 0.434$, and $2.372 \pm 0.133$ fold control groups levels after $1 \mathrm{~d}$ of intermittent stretching. The ALP, COL I, OCN, and osterix mRNA levels were $2.046 \pm 0.129,3.904 \pm 0.120,5.588 \pm 0.420$, and $3.570 \pm 0.420$ fold control group levels after $3 \mathrm{~d}$ of intermittent stretching and the ALP, COL I, OCN, and osterix mRNA levels were $4.705 \pm 0.346,5.106 \pm 0.213,9.076 \pm 0.205$, and $7.031 \pm 0.196$ fold control levels after $5 \mathrm{~d}$ of intermittent stretching. The changes in mRNA levels over time were statistically significant for every osteogenesis gene $\left({ }^{*} P<0.05\right.$ or $\left.{ }^{* *} P<0.01\right)$ (Fig. 4). The results showed that mRNA expression of bone-related genes, ALP, COL I, OCN, and osterix, increased from day 1 to day 5 in varying proportions. The increasing expression of ALP, COL I, OCN, and osterix mRNA levels were similar over the studied time-points.

Intermittent stretching induced the osteogenic differentiation of BMMSCs via osterix

The aforementioned data indicate that the expression of osterix mRNA, increased on day 1 and lasted until day 5 (Fig. 4D). The following experiment was conducted to determine whether osterix is involved in osteogenic differentiation of mouse BMMSCs attributable to 

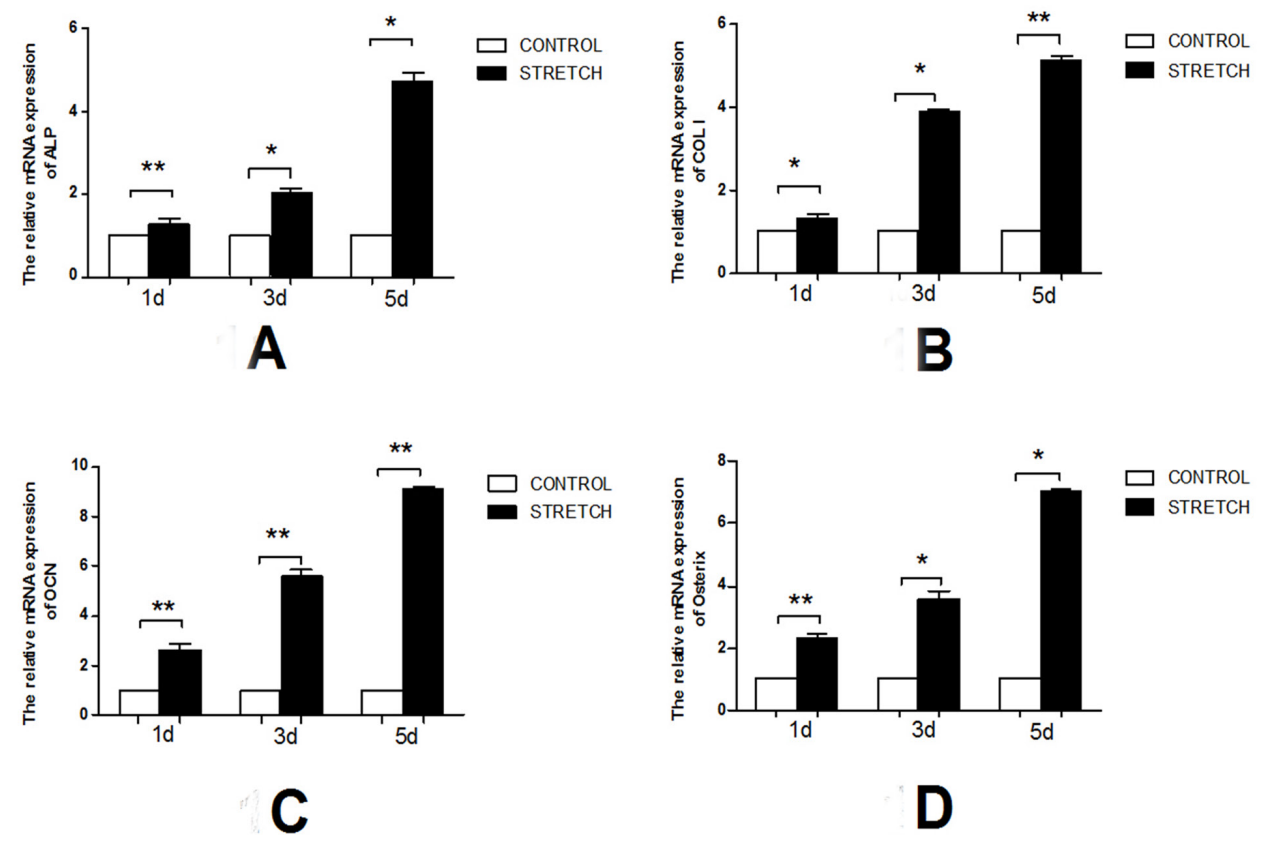

Fig. 4. Osteogenic differentiation of BMMSCs induced by intermittent stretching. BMMSCs underwent intermittent stretching for 1,3 , and 5 days. The control groups were prepared at the same time-points without intermittent stretch stimulation. RT- PCR analysis of (A) ALP, (B) COL I, (C) OCN, and (D) osterix mRNA in BMMSCs after intermittent stretch stimulation [mean \pm SD. $\mathrm{n}=3$ (per group), ${ }^{*} P<0.05,{ }^{* *} P<0.01$ ] .
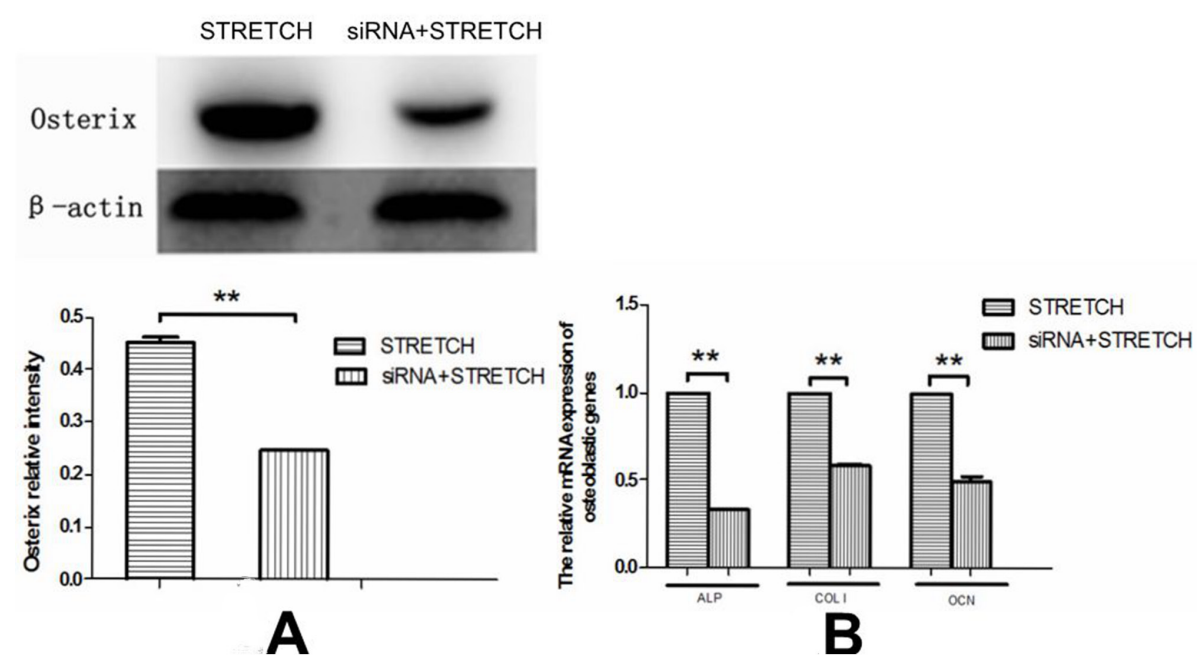

Fig. 5. Osteogenic differentiation of BMMSCs subjected to intermittent stretching via osterix. (A) Osterix knockdown was confirmed after transfection by Western blot following transfection. (B) Real-time PCR analysis of ALP, COL I, and OCN mRNA in BMMSCs on day 1 of intermittent stretch stimulation (also day 1 post-transfection) [mean \pm SD. $n=3$ (per group), ${ }^{*} P<0.05,{ }^{* *} P<0.01$ ].

mechanical stretching. BMMSCs were transfected using with osterix-siRNA, and then the expression of ALP, COL I, and OCN mRNA was determined by RT-PCR and subsequently Western blot analyses following $24 \mathrm{~h}$ of stretching. Western blot data demonstrates that the relative expression of osterix protein between siRNA+stretch group and control group (non-silencing siRNA+stretch) to be $0.452 \pm 0.016$ and $0.246 \pm 0.003$, respectively (the value 


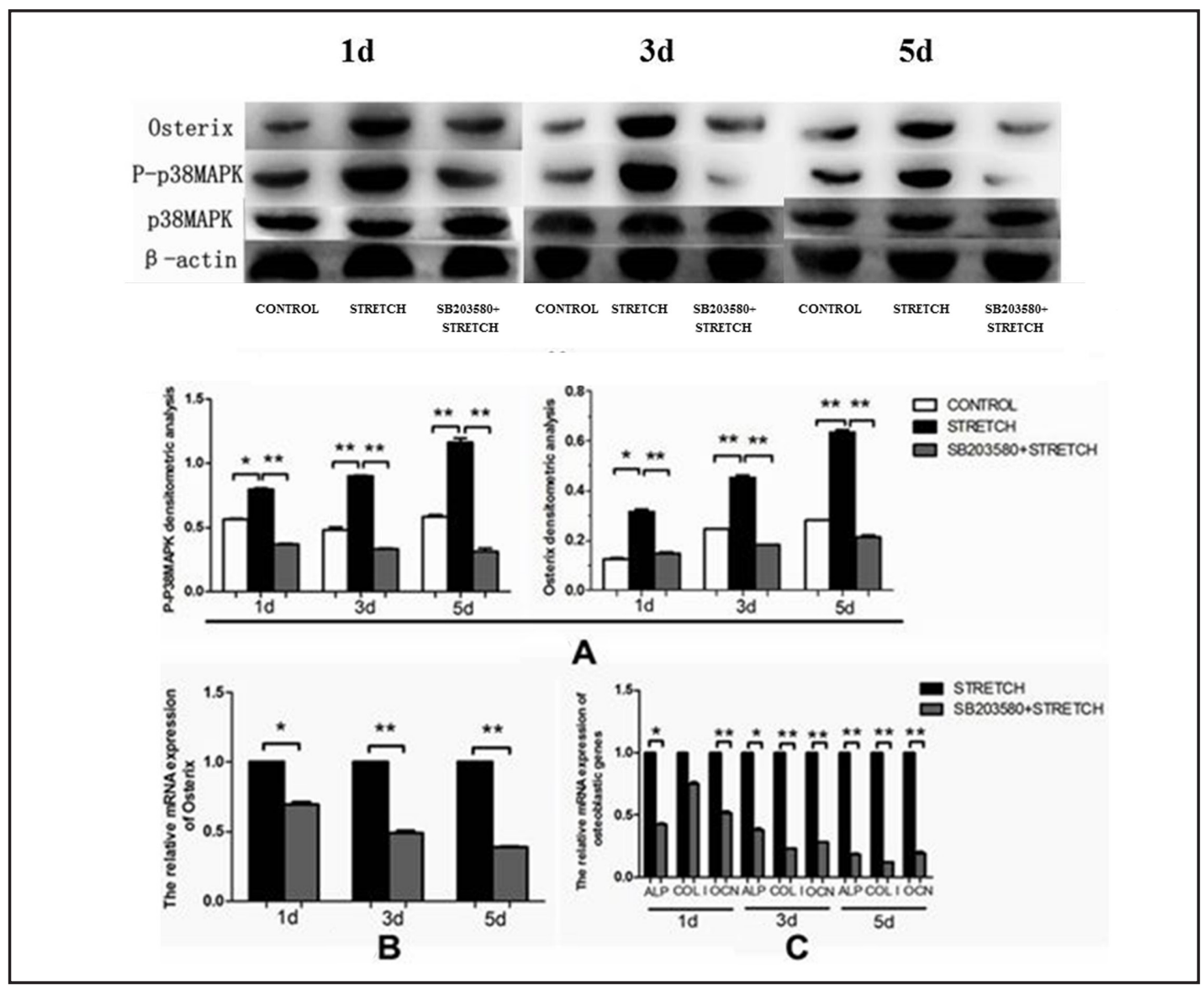

Fig. 6. Osteogenic differentiation of BMMSCs subjected to intermittent stretching via the p38MAPK-osterix signaling pathway. BMMSCs were pre-treated with SB203580 for $1 \mathrm{~h}$ prior to intermittent stimulation for 1, 3 , and 5 days. Control groups were prepared at the same points in time without intermittent stretch stimulation. (A) Western blot analysis of P-p38MAPK and osterix protein in BMMSCs on days 1, 3, and 5. Real-time PCR analysis of (C) ALP, (C) COL I, (C) OCN, and (B) osterix mRNA on days 1, 3, and 5 [mean \pm SD. $n=3$ (per group), $\left.{ }^{*} P<0.05,{ }^{* *} P<0.01\right]$.

is the osterix to $\beta$-actin ratio). The osterix protein levels were significantly lower in the siRNA+stretch group than in the control group $(* * P<0.01)$ (Fig. 5A). RT- PCR results showed ALP, COL I, and OCN mRNA levels to be $0.327 \pm 0.012,0.587 \pm 0.014$, and $0.493 \pm 0.053$ fold compared to those of the control groups, respectively, after $1 \mathrm{~d}$ of intermittent stretching. The mRNA levels of ALP, COL I, and OCN in the siRNA+stretch group decreased more than in the control group $\left({ }^{*} P<0.05\right.$ or ${ }^{* *} P<0.01$ ) (Fig. 5B). These results suggest that osterix silencing inhibited osteogenic differentiation of BMMSCs induced by intermittent stretching.

Intermittent stretching induced the osteogenic differentiation of BMMSCs via p38MAPKosterix signaling pathway

The protein levels of P-p38MAPK and osterix were investigated among the control, intermittent stretch group, and SB230850+ intermittent stretching group (days 1, 3, and 5). The Western blot results demonstrated that, under intermittent stretch stimulation, the P-p38MAPK and osterix protein levels of BMMSCs increased significantly on day 1 and continued to increase relative to controls until day 5. After 1 day of intermittent stretching, the protein expression of P-p38MAPK among three groups was $0.565 \pm 0.008,0.798 \pm 0.017$, and $0.371 \pm 0.008$, respectively. The protein expression of osterix among three groups was $0.125 \pm 0.004,0.318 \pm 0.014$, and $0.150 \pm 0.003$, respectively. After 3 days of intermittent stretching, the protein expression of P-p38MAPK among three groups was $0.579 \pm 0.043$, 
$0.905 \pm 0.007$, and $0.332 \pm 0.010$, respectively. The protein expression of osterix among three groups was $0.246 \pm 0.003,0.452 \pm 0.015$, and $0.182 \pm 0.003$, respectively. After 5 days of intermittent stretching, the protein expression of P-p38MAPK among three groups was $0.587 \pm 0.023,1.165 \pm 0.057$, and $0.314 \pm 0.040$, respectively. The protein expression of osterix in the three groups was $0.281 \pm 0.004,0.635 \pm 0.019$, and $0.215 \pm 0.011$, respectively. The protein expression of P-p38MAPK and osterix among three groups was significantly different $\left({ }^{*} P<0.05\right.$ or $\left.{ }^{* *} P<0.01\right)$. Among the three groups, P-p38MAPK activity was more pronounced in the intermittent stretching group than in the control group at several points in time. When P-p38MAPK activity was blocked, protein levels decreased over time. The osterix protein levels had similar expression tendencies as P-p38MAPK (Fig. 6A).

The levels of ALP, COL I, OCN, and osterix mRNA were investigated under both intermittent stretching and SB230850+ intermittent stretching conditions (days 13 , and 5). Real-time PCR results showed there to be decreased expression of ALP, COL I, OCN, and osterix mRNA in the SB230850+intermittent stretching group than in the intermittent stretching group. When P-p38MAPK activity was blocked for 1 day, the ALP, COL I, OCN, and osterix mRNA levels of the intermittent stretching group were $0.427 \pm 0.014,0.753 \pm 0.023,0.515 \pm 0.024$, and $0.697 \pm 0.025$ fold, respectively, compared to those of the SB230850+intermittent stretching group. When P-p38MAPK activity was blocked for 3 days, the ALP, COL I, OCN, and osterix mRNA levels of the intermittent stretching group were $0.380 \pm 0.023,0.233 \pm 0.009$, $0.280 \pm 0.011$, and $0.491 \pm 0.029$ fold, respectively, those of the SB230850+intermittent stretching group. When P-p38MAPK activity was blocked for 5 days, the ALP, COL I, OCN, and osterix mRNA of the intermittent stretching group were $0.189 \pm 0.010,0.123 \pm 0.005$, $0.197 \pm 0.014$, and $0.388 \pm 0.012$ fold, respectively, those of the SB230850+intermittent stretching group. The differences (except COL I mRNA on day 1) were all statistically significant $\left({ }^{*} P<0.05\right.$ or ${ }^{* *} P<0.01$ ) (Fig. 6B and 6C). These results indicate that the p38MAPKosterix signaling pathway contributes to the process of osteogenic differentiation of BMMSCs induced by intermittent stretching.

\section{Discussion}

Bone is a metabolically active and highly organized organ. It can sense and adapt to mechanical stimulation and keep bone resorption and reconstruction in a state of equilibrium. Osteogenic differentiation of BMMSCs induced by mechanical stretch stimulation is the key cytological basis of distraction osteogenesis, orthodontic tooth movement, and the formation of new bone during fracture healing. This phenomenon has been reported to have wide application in a variety of clinical settings including craniofacial surgery, temporomandibular joint disorders, and maxillofacial trauma [15-17]. The mitogen-activated protein kinase (MAPK) family, which includes extracellular signal regulated kinase 1/2 (ERK1/2), c-Jun $\mathrm{N}$-terminal kinase (JNK)/stress activated protein kinase (SAPK), and the p38MAPK pathway, can transmit stimulation from the extracellular space to the intracellular space. These MAPK family members are also involved in the physiological processes of cell growth and differentiation $[18,19]$. Specifically, the p38MAPK signaling pathway has a crucial role in the inflammatory response, regulating the cell cycle, cell differentiation and apoptosis [20,21]. Previous studies have shown that the p38MAPK signaling pathway has a close relationship with osteogenic differentiation of BMMSCs [2-6].

It has been demonstrated that the roles of BMP9, 8-prenylnaringenin tigogenin, and other substances can activate the p38MAPK signaling pathway and promote osteogenic differentiation of BMMSCs [2, 4-6]. This collectively shows that the p38MAPK signaling pathway may play an important role in the osteogenic differentiation of BMMSCs. The p38MAPK signaling pathway has been proven to be stress-sensitive in a variety of other relevant biological systems [22-24]. However, the relationship between osteogenic differentiation of BMMSCs induced by mechanical stretch stimulation and the p38MAPK signaling pathway is still controversial. Data derived by Simmons et al. demonstrated that 0.25

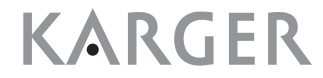


$\mathrm{Hz}$ and 3\% (30000 $\mu$ strain) stretching can quickly activate the p38MAPK signaling pathway without causing obvious changes in the level of bone differentiation. However, it can increase levels of bone differentiation using p38MAPK-specific inhibitors. The p38MAPK signaling pathway was found to have an opposite effect on the osteogenic differentiation of hMSCs induced by stretching [25]. However, Zhang et al. found that the levels of bone differentiation were not significantly affected by the p38MAPK-specific inhibitor, SB203580, after the rat BMMSCs were subjected to $1 \mathrm{~Hz} 10 \%$ (100000 $\mu$ strain) stretching. They concluded that the p38MAPK signaling pathway is not involved in osteogenic differentiation of rat BMMSCs [26]. Qi et al. found that $2000 \mu$ stretching can promote osteogenic differentiation of BMMSCs [27]. It has been reported that stress with $0.8 \%(8000 \mu$ strain) stretching can promote osteogenic differentiation of ST2, the stromal cell line, while $10 \%$ and $15 \%(150000 \mu$ strain) stretching inhibits it [1]. Thus, in the current study, mouse C57BL/6J BMMSCs were subjected to stretching at $0.5 \mathrm{~Hz}$ and $0.8 \%$ for 30 min twice daily. P-p38MAPK activity was found to increase over time under intermittent stretch stimulation conditions. Following inhibition by the addition of p38MAPK-specific inhibitor, SB203580, P-p38MAPK activity decreased over time. The trends in the expression of osteogenesis-related genes (ALP, COL I, and OCN) were consistent with the expression of P-p38MAPK. This demonstrates that the p38MAPK pathway is involved in the osteogenic differentiation of BMMSCs.

Osterix knockout mice eventually succumb due to breathing difficulties and histological results have shown that there is no mineralization reaction in mice embryos. Neither endochondral ossification nor membrane ossification was completed [28-30]. ALP, OCN, and COL I are typically used in identification of osteogenic differentiation in cells [31]. The expression of genes related to osteogenic differentiation (ALP and OCN) is significantly less pronounced in osterix knockout mice [32]. OCN is a highly specific marker of osteogenic differentiation, and its expression was decreased, indicating that osteogenic differentiation and maturation were blocked. This suggests that osterix has an important role in the regulation of osteogenic differentiation. Overexpression of osterix was found to induce osteogenic differentiation in mouse embryonic stem cells [33], BMMSCs [34], and adiposederived stem cells [35]. In this manner, they may facilitate the understanding of osteogenic differentiation and bone-related diseases by elucidating the mechanisms of expression and regulation of osterix.

Studies have shown that mechanical stimulation can induce osterix expression. Zhao et al. demonstrated that when periodontal ligament cells are stimulated by centrifugal force, the levels of osterix mRNA and protein increases in a time-dependent manner and that overexpression of osterix can significantly promote osteogenic differentiation among periodontal ligament cells [11]. Furthermore, Kang et al. combined ultrasonic stimulation with mechanical stretching on osteoblast precursor cells to study their effects on the proliferation and differentiation of osteoblast precursor cells. This mechanical stretching was found to increase the expression of osterix and then promote osteogenic differentiation through the osteoblast precursor cell pathway [9]. Hence, osterix is closely related to osteogenic differentiation induced by mechanical stimulation.

Prior to this current study, the effects of osterix in the osteogenic differentiation of BMMSCs induced by mechanical stretching were still unclear. The current study showed that the mRNA levels of osterix and osteogenesis-related genes (ALP, COL I, and OCN) increased under intermittent stretch stimulation conditions. However, knockdown of osterix was found to down-regulate the mRNA expression levels of osteogenesis-related genes (ALP, COL $\mathrm{I}$, and OCN) in BMMSCs. This indicates that osterix plays an important role in the osteogenic differentiation of BMMSCs induced by mechanical stretching.

The purpose of this study was to elucidate the effects of the p38MAPK signal pathway and osterix in the process of osteogenic differentiation of BMMSCs induced by mechanical stretching and the relationship between the two. Under intermittent stretch stimulation conditions, the expression activity of osterix was decreased accordingly when the p38MAPKspecific inhibitor, SB203580, was used to block the activity of P-p38MAPK. The activity and expression of osteogenesis-related genes (ALP, COL I, and OCN) also decreased. These 


\section{Cellular Physiology Cell Physiol Biochem 2015;36:1015-1025 \begin{tabular}{l|l} 
Dol: 10.1159/000430275 & $\begin{array}{l}\text { 2015 s. Karger AG, Basel } \\
\text { www.karger.com/cpb }\end{array}$ \\
\hline
\end{tabular}

results confirm that the p38MAPK signaling pathway is indispensable to osterix and that blocking the p38MAPK signaling pathway inhibits osteogenic differentiation of BMMSCs. In addition, proper mechanical stretching was found to promote osteogenic differentiation of mouse BMMSCs via the p38MAPK-osterix signaling pathway, and the p38MAPK-osterix pathway plays an important role in the control of osteogenesis-related gene expression. The distraction frequency is an important factor for determining new bone formation during distraction osteogenesis. Usually it is performed 2-4 times every day clinically. Research has shown that high frequency and low stretch power were associated with more callus formation. The mechanical stimulation of $0.8 \%$ (8000 $\mu$ strain) stretching lies within a physiological range at the cellular level [36]. Hence the data presented within our study is also consistent with clinical tension models including distraction osteogenesis. Our present study may provide some insight and may further the understanding of osteogenic differentiation of BMMSCs induced by biomechanical stimulation. However, thorough examination into this phenomenon is required to confirm developmental trends and develop this information towards clinical utility.

\section{Disclosure Statement}

The authors declare that they have no competing interests.

\section{Acknowledgments}

This work was supported by Sci-Tech Research Projects of Huangdao District of Qingdao City (2014-1-84).

\section{References}

1 Koike M, Shimokawa H, Kanno Z, Ohya K, Soma K: Effects of mechanical strain on proliferation and differentiation of bone marrow stromal cell line ST2. J Bone Miner Metab 2005;23:219-225.

2 Ming L, Ge B, Wang M, Chen K: Comparison between 8-prenylnarigenin and narigenin concerning their activities on promotion of rat bone marrow stromal cells' osteogenic differentiation in vitro. Cell Proliferation 2012;45:508-515.

3 Ming LG, Zhou J, Cheng GZ, Ma HP, Chen KM: Osthol, a coumarin isolated from common cnidium fruit, enhances the differentiation and maturation of osteoblasts in vitro. Pharmacology 2011;88:33-43.

4 Xu DJ, Wang J, Weng YG: Smads, p38 and ERK1, 2 are involved in BMP9-induced osteogenic differentiation of C3H10T1, 2 mesenchymal stem cells. BMB Rep 2012;45:247-252.

5 Zhao Y, Song T, Wang W, Wang J, He J, Wu N, Tang M, He B, Luo J: P38 and ERK1/2 MAPKs act in opposition to regulate BMP9-induced osteogenic differentiation of mesenchymal progenitor cells. PLoS One 2012; 7:e43383.

6 Zhou H, Yang X, Wang N, Zhang Y, Cai G: Tigogenin inhibits adipocytic differentiation and induces osteoblastic differentiation in mouse bone marrow stromal cells. Mol Cell Endocrinol 2007;270:17-22.

7 Yang L, Cheng P, Chen C, He HB, Xie GQ Zhou HD, Xie H, Wu XP, Luo XH: miR-93/Sp7 function loop mediates osteoblast mineralization. J Bone Miner Res 2012;27:1598-1606.

8 Chen D, Li Y, Zhou Z, Xing Y, Zhong Y, Zou X, Tian W, Zhang C: Synergistic inhibition of Wnt pathway by HIF$1 \alpha$ and osteoblast-specific transcription factor osterix (Osx) in osteoblasts. PLoS One 2012;7:e52948.

9 Kang KS, Lee SJ, Lee H, Moon W, Cho DW: Effects of combined mechanical stimulation on the proliferation and differentiation of pre-osteoblasts. Exp Biol Med 2011;43:367-373.

10 Zhang C, Li J, Zhang L, Zhou Y, Hou W, Quan H, Li X, Chen Y, Yu H: Effects of mechanical vibration on proliferation and osteogenic differentiation of human periodontal ligament stem cells. Arch Oral Biol 2012;57:1395-1407.

11 Zhao Y, Wang C, Li S, Song H, Wei F, Pan K, Zhu K, Yang P, Tu Q, Chen J: Expression of Osterix in mechanical stress-induced osteogenic differentiation of periodontal ligament cells in vitro. Eur J Oral Sci 2008;116:199-206.

12 Soleimani M, Nadri S: A protocol for isolation and culture of mesenchymal stem cells from mouse bone marrow. Nat Protoc 2009;4:102-106. 


\section{Cellular Physiology Cell Physiol Biochem 2015;36:1015-1025 \begin{tabular}{l|l|l} 
DOI: 10.1159/000430275 & C 2015 S. Karger AG, Basel
\end{tabular} and Biochemistry Published online: June 16, 2015

13 Zhu H, Guo ZK, Jiang XX, Li H, Wang XY, Yao HY, Zhang Y, Mao N: A protocol for isolation and culture of mesenchymal stem cells from mouse compact bone. Nat Protoc 2010;5:550-560.

14 Govey PM, Loiselle AE, Donahue HJ: Biophysical regulation of stem cell differentiation. Curr Osteoporos Rep 2013;11:83-91.

15 Ellegaard M, Kringelbach T, Syberg S, Petersen S, Beck Jensen JE, Brüel A, Jørgensen NR, Schwarz P: The effect of PTH (1-34) on fracture healing during different loading conditions. J Bone Miner Res 2013;28:2145-2155.

16 Luther F, Layton S, McDonald F: Orthodontics for treating temporomandibular joint (TMJ) disorders. Cochrane Database Syst Rev 2010;7:CD006541.

17 Rachmiel A, Emodi O, Gutmacher Z, Blumenfeld I, Aizenbud D: Oral and dental restoration of wide alveolar cleft using distraction osteogenesis and temporary anchorage devices. J Craniomaxillofac Surg 2013;41:728-734.

18 Suzuki A, Guicheux J, Palmer G, Miura Y, Oiso Y, Bonjour JP, Caverzasio J: Evidence for a role of p38 MAP kinase in expression of alkaline phosphatase during osteoblastic cell differentiation. Bone 2002;30:91-98.

19 Xiao G, Jiang D, Thomas P, Benson MD, Guan K, Karsenty G, Franceschi RT: MAPK pathways activate and phosphorylate the osteoblast-specific transcription factor, Cbfa1. J Biol Chem 2000;275:4453-4459.

20 Coulthard LR, White DE, Jones DL, McDermott MF, Burchill SA: p38MAPK: stress responses from molecular mechanisms to therapeutics. Trends Mol Med 2009;15:369-379.

21 Yang F, Chen H, Liu Y, Yin K, Wang Y, Li X, Wang G, Wang S, Tan X, Xu C, Lu Y, Cai B. Doxorubicin caused apoptosis of mesenchymal stem cells via p38, JNK and p53 pathway. Cell Physiol Biochem. 2013;32:10721082.

22 Du QC, Zhang DZ, Chen XJ, Lan-Sun G, Wu M, Xiao WL: The Effect of p38MAPK on Cyclic Stretch in Human Facial Hypertrophic Scar Fibroblast Differentiation. PLoS One 2013;8:e75635.

23 Li F, Yan X, Wang Q, Qu Z, Yuan X, Guo J: Effect of p38 mitogen-activated protein kinase signaling pathway during stretch-induced differentiation process of C2C12 myoblasts. Hua Xi Kou Qiang Yi Xue Za Zhi 2012;30:574-578.

24 Wang L, Li JY, Zhang XZ, Liu L, Wan ZM, Li RX, Guo Y: Involvement of p38MAPK/NF- $K B$ signaling pathways in osteoblasts differentiation in response to mechanical stretch. Ann Biomed Eng 2012;40:1884-1894.

25 Simmons CA, Matlis S, Thornton AJ, Chen S, Wang CY, Mooney DJ: Cyclic strain enhances matrix mineralization by adult human mesenchymal stem cells via the extracellular signal-regulated kinase (ERK1/2) signaling pathway. J Biomech 2003;236:1087-1096.

26 Zhang P, Wu Y, Dai Q Fang B, Jiang L: p38-MAPK signaling pathway is not involved in osteogenic differentiation during early response of mesenchymal stem cells to continuous mechanical strain. Mol Cell Biochem 2013;378:19-28.

27 Qi MC, Zou SJ, Han LC, Zhou HX, Hu J. Expression of bone-related genes in bone marrow MSCs after cyclic mechanical strain: implications for distraction osteogenesis. Int J Oral Sci 2009;1:143-150.

28 Xu DJ, Wang J, Weng YG: Smads, p38 and ERK1, 2 are involved in BMP9-induced osteogenic differentiation of C3H10T1, 2 mesenchymal stem cells. BMB Rep 2012;45:247-252.

29 Nakashima K, de Crombrugghe B: Transcriptional mechanisms in osteoblast differentiation and bone formation. Trends Genet 2003;19:458-466.

30 Nakashima K, Zhou X, Kunkel G, Zhang Z, Deng JM, Behringer RR, de Crombrugghe B: The novel zinc fingercontaining transcription factor osterix is required for osteoblast differentiation and bone formation. Cell 2002;108:17-29.

31 Chen Q, Liu W, Sinha KM, Yasuda H, de Crombrugghe B: Identification and characterization of microRNAs controlled by the osteoblast-specific transcription factor Osterix. PLoS One 2013;8:e58104.

32 Baek WY, Lee MA, Jung JW, Kim SY, Akiyama H, de Crombrugghe B, Kim JE: Positive regulation of adult bone formation by osteoblast-specific transcription factor osterix. J Bone Miner Res 2009;24: 1055-1065.

33 Tai G, Polak JM, Bishop AE, Christodoulou I, Buttery LD: Differentiation of osteoblasts from murine embryonic stem cells by overexpression of the transcriptional factor osterix. Tissue Eng 2004;10:14561466.

34 Tu Q Valverde P, Chen J: Osterix enhances proliferation and osteogenic potential of bone marrow stromal cells. Biochem Biophys Res Commun. 2006;341:1257-1265.

35 Wu L, Wu Y, Lin Y, Jing W, Nie X, Qiao J, Liu L, Tang W, Tian W: Osteogenic differentiation of adipose derived stem cells promoted by overexpression of osterix. Mol Cell Biochem 2007;301:83-92.

36 Meyer U, Meyer T, Wiesmann HP, Stratmann U, Kruse-Lösler B, Maas H, Joos U: The effect of magnitude and frequency of interfragmentary strain on the tissue response to distraction osteogenesis. J Oral Maxillofac Surg 1999;57:1331-1339. 